\title{
Allelic variation in 5-HTTLPR and the effects of citalopram on the emotional neural network
}

\author{
Yina Ma, Bingfeng Li, Chenbo Wang, Wenxia Zhang, Yi Rao and Shihui Han
}

\section{Background}

Selective serotonin reuptake inhibitors (SSRIS), such as citalopram, which selectively block serotonin transporter (5-HTT) activity, are widely used in the treatment of depression and anxiety disorders. Numerous neuroimaging studies have examined the effects of SSRIS on emotional processes. However, there are considerable inter-individual differences in SSRI effect, and a recent meta-analysis further revealed discrepant effects of acute SSRI administration on neural responses to negative emotions in healthy adults.

\section{Aims \\ We examined how a variant of the serotonin-transporter polymorphism (5-HTTLPR), which affects the expression and function of 5-HTT, influenced the acute effects of an SSRI (citalopram) on emotion-related brain activity in healthy adults.}

\section{Method}

Combining genetic neuroimaging, pharmacological technique and a psychological paradigm of emotion recognition, we scanned the short/short (s/s) and long/long (I/l) variants of 5-HTTLPR during perception of fearful, happy and neutral facial expressions after the acute administration of an SSRI (i.e. $30 \mathrm{mg}$ citalopram administered orally) or placebo administration.

\section{Results}

We found that 5-HTTLPR modulated the acute effects of citalopram on neural responses to negative emotions. Specifically, relative to placebo, citalopram increased amygdala and insula activity in I/I but not $\mathrm{s} / \mathrm{s}$ homozygotes during perception of fearful faces. Similar analyses of brain activity in response to happy faces did not show any significant effects.

\section{Conclusions}

Our combined pharmacogenetic and functional imaging results provide a neurogenetic mechanism for discrepant acute effects of SSRIS.

\section{Declaration of interest}

None.

\section{Copyright and usage}

(c) The Royal College of Psychiatrists 2015.
Selective serotonin reuptake inhibitors (SSRIs) are a class of antidepressants and have been used to treat mood disorders, such as depression and anxiety disorder. Selective serotonin reuptake inhibitors such as citalopram selectively block serotonin transporter (5-HTT) activity, ${ }^{1}$ inhibit the reuptake of serotonin (5-HT) into the presynaptic cell and lead to increasing extracellular levels of $5 \mathrm{HT}^{2}$ Although clinical trials have shown that SSRIs typically have a delay of several weeks in the onset of their clinical effects, SSRI effects can occur soon after their administration. ${ }^{3}$ The early changes in emotional processing that occur after the acute administration of an SSRI contribute to later mood improvements. ${ }^{4,5}$ Therefore, understanding the neural mechanisms underlying the acute effects of SSRIs is important for decisions about long-term treatment. However, recent neuroimaging studies have reported inconsistent acute effects of SSRIs on neural responses in emotion-related brain regions such as the amygdala, whose hyperactivity has been suggested as an endophenotype of anxiety disorder and major depression.,

Twelve functional magnetic resonance imaging (fMRI) studies have examined the acute effects of SSRIs (single SSRI administration) on neural responses to negative emotions, while eight fMRI studies have examined acute effects of SSRI administration on neural response to positive emotions. Among these studies, four reported increased amygdala response ${ }^{8-11}$ whereas eight reported decreased amygdala response to negative emotions ${ }^{11-18}$ after SSRI $v$. placebo administration. Two studies reported increased amygdala response to positive emotions, ${ }^{10,19}$ whereas six studies did not show SSRI effects on amygdala response to positive emotions. ${ }^{9,10,14-16,19}$ Moreover, our recent meta-analysis ${ }^{20}$ revealed that the acute effects of antidepressants on positive emotions did not show any convergent activation in healthy adults. Acute antidepressant administration showed discrepant effects on neural responses to negative emotions. Specifically, single antidepressant administration led to convergent increases as well as decreases in a similar neural network underlying negative emotions. Although age, gender or disease conditions may contribute to individual differences in psychometric outcome and discrepant SSRI effects on amygdala response, the genetic structure of individuals also affects drug responses. ${ }^{21}$ A sodiumdependent serotonin-transporter-linked polymorphic region (5-HTTLPR), which influences the expression and function of 5 -HTT, ${ }^{22,23}$ may be implicated in SSRI efficacy. For example, major depression patients with the long (l) allele of 5-HTTLPR showed better responses to SSRI treatment compared to homozygotes for the short variant $(\mathrm{s} / \mathrm{s})$ of 5 -HTTLPR. ${ }^{24,25}$ However, it remains unknown whether and how 5-HTTLPR polymorphism affects the acute effects of SSRIs on brain activity.

\section{Method}

\section{Experimental design}

We combined genetic neuroimaging, pharmacological technique and a psychological paradigm of emotion recognition to provide a better understanding of the neurogenetic mechanism of the acute effects of SSRIs on emotions. Specifically, we examined whether 5-HTTLPR modulates the acute effects of SSRIs on neural response to emotional facial expressions. Using a double-blind, within-subject, counterbalanced design (Fig. 1), we scanned s/s and $1 / 1$ homozygotes during perception of fearful, happy and neutral facial expressions using fMRI after administration of a single oral dose of a SSRI (30 mg citalopram) and a matched 

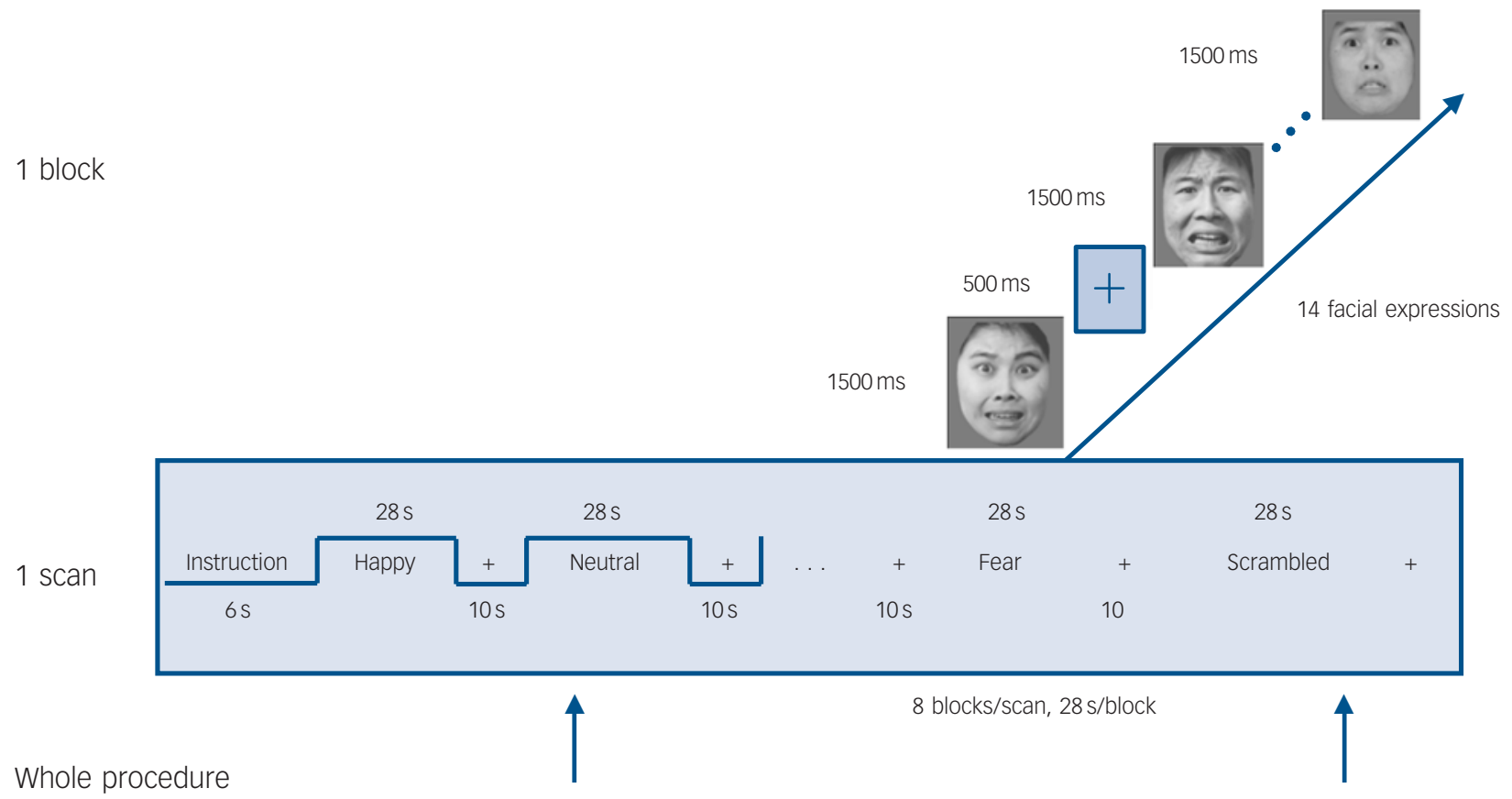

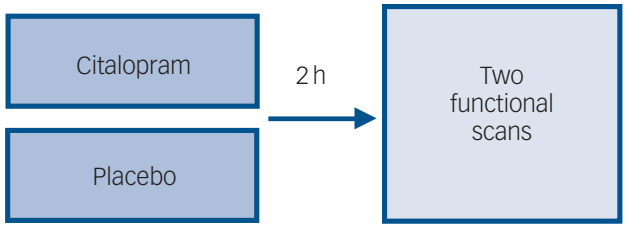

Single dose

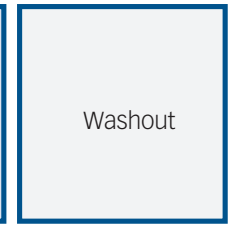

$\geqslant 7$ days

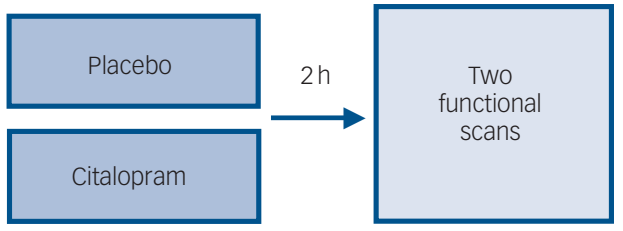

Single dose

Fig. 1 Experimental procedure. Each individual participated in two fMRI sessions separated by a 7-day washout. In each session, functional scanning was conducted $2 \mathrm{~h}$ after citalopram or placebo administration. A block design was used during scanning. There were two functional scans lasting $334 \mathrm{~s}$ in each (citalopram or placebo) session. Each functional scan consisted of eight blocks (two blocks of each stimulus category) of 14 stimuli (12 faces and two repeats) from the same stimulus category. Each face stimulus was presented for $1500 \mathrm{~ms}$; participants were then asked to keep their gaze on a fixation cross for $500 \mathrm{~ms}$. Two successive blocks were separated by a $10 \mathrm{~s}$ fixation cross. fMRI: functional magnetic resonance imaging.

placebo tablet with at least a 7-day washout period (at least 7 days apart, ranging from 7 to 29 days, mean $=14.3$ days, s.d. $=6.7$ ). Given that $1 / 1$ compared with s/s homozygotes have been shown to exhibit increased 5-HTT expression and 5-HT uptake, ${ }^{26}$ and a greater increase in $5 \mathrm{HT}$ neurotransmission after acute blockade of 5-HT reuptake, ${ }^{27}$ blocking 5-HTT activity with citalopram may produce stronger effects on brain activity in $1 / 1$ than $s / s$ homozygotes. Thus, we predicted greater SSRI effects on the neural responses to emotional facial expressions in $1 / 1$ than $s / s$ homozygotes.

\section{Participants}

Fifty Chinese men participated in this study as paid volunteers. We recruited only men to avoid any potential gender difference in citalopram effects. We used polymerase chain reaction to determine the genotypes of 5-HTTLPR. (See online supplementary material for DNA isolation and analysis.) Two $1 / 1$ and two s/s homozygotes were excluded from the data analysis due to excessive head movement during scanning (exclusion criterion: $6 \mathrm{~mm}$ head motion or $3^{\circ}$ for head rotation). Thus $23 \mathrm{~s} / \mathrm{s}$ (18-23 years, mean $=19.4$, s.d. $=1.8)$ and $231 / 1$ homozygotes $(18-23$ years, mean $=18.6$, s.d. $=1.4$ ), who did not differ significantly in age $(t(44)=1.296, P=0.202)$, were included in the data analysis. All participants were right-handed and had normal or correctedto-normal vision. All participants had no history of cardiac, hepatic, renal, pulmonary, neurological, psychiatric or gastrointestinal disorders, medication/drug use, and no family history of major depression or bipolar affective disorder. The study protocol was approved by the local ethics committee of the Department of Psychology at Peking University. Written informed consent was obtained prior to the study from all participants.

\section{General procedure}

We used a $2 \times 2 \times 2$ factorial design with emotion (fearful $v$. neutral faces, or happy $v$. neutral faces) and treatment (citalopram $v$. placebo) as the between-subjects variables and genotype (s/s $v .1 / 1$ genotype) as the between-subjects variable. The order of citalopram and placebo treatment was counterbalanced within each genotype group and across the s/s and 1/l genotype groups. We used a single oral administration of $30 \mathrm{mg}$ citalopram, which is within the dose range $(20-60 \mathrm{mg})$ and has been used in previous studies. ${ }^{28,29}$ We administered a single dose to examine the acute effects of SSRIs. Pharmacokinetic and plasma hormone concentration studies have shown that, in men, citalopram plasma concentration reaches a peak around $2 \mathrm{~h}^{30,31}$ Thus, functional scanning was conducted about $2 \mathrm{~h}$ after citalopram or placebo administration when citalopram reached its peak effect. During the waiting period, participants sat on a comfortable couch, resting or reading. A researcher explained the task and procedure to participants and participants practised a few trials to become 
familiar with the task 30 min before scanning. Before taking citalopram or the placebo, we evaluated each participant's harm-avoidance tendency using the harm avoidance subscale from the Tridimensional Personality Questionnaire. ${ }^{32}$ At the beginning and end of the citalopram or placebo session, we asked participants to complete the Positive and Negative Affect Scale ${ }^{33}$ to monitor their mood changes.

\section{Functional imaging task}

Four categories of facial expressions (fearful, happy, neutral and scrambled; 24 images in each category, half male models and half female models, from the Chinese Facial Affective Picture System $)^{34}$ were used during scanning. Scrambled faces were made by segmenting each face image $(300 \times 336$ pixels $)$ into $50 \times 56$ square grids $(6 \times 6$ pixels $)$ that were then randomly arranged. This rearranged picture was then masked by a stencil of the original face shape, resulting in a pixellated face on a grey background. Stimuli used during the scanning were delivered through a LCD projector onto a rear projection screen (SINORAD SA-9900). Each stimulus subtended a visual angle of $3.85^{\circ} \times 4.31^{\circ}$ (width $\times$ height) at a viewing distance of $83 \mathrm{~cm}$. Participants completed a typical repetition-detection task by responding to repeated facial expressions via a button press (Fig. 1). After scanning, participants were asked to rate the emotion intensity of 12 fearful and 12 happy faces randomly selected from the two categories on an 11-point Likert scale $(0=$ not at all fearful/happy; $10=$ extremely fearful $/$ happy).

\section{Imaging parameters}

We acquired images of the brain using a Siemens 3-Tesla Trio MRI scanner at the Beijing MRI Center for Brain Research. We obtained blood oxygenation level-dependent gradient-echo echo-planar images using a 12-channel head coil $(64 \times 64 \times 32$ matrix with $3.44 \times 3.44 \times 5.0 \mathrm{~mm}$ spatial resolution, repetition time $(\mathrm{TR})=2000 \mathrm{~ms}$, echo time $(\mathrm{TE})=30 \mathrm{~ms}$, flip angle $=90^{\circ}$, field of view $=24 \times 24 \mathrm{~cm}$ ) while the participants performed the repetition-detection task. We subsequently acquired a highresolution, $\mathrm{T}_{1}$-weighted structural image $(256 \times 256 \times 144$ matrix with a spatial resolution of $1 \times 1 \times 1.33 \mathrm{~mm}, \mathrm{TR}=2530 \mathrm{~ms}$, $\mathrm{TE}=3.37 \mathrm{~ms}$, inversion time $(\mathrm{TI})=1100 \mathrm{~ms}$, flip angle $\left.=7^{\circ}\right)$.

\section{Imaging analysis}

\section{Preprocessing}

We used the Statistical Parametric Mapping software, version 8 (Wellcome Trust Centre for Neuroimaging; http://www.fil.ion. ucl.ac.uk/spm) to analyse the functional image data; we analysed data using the general linear model for a block design. We realigned the functional images within and across runs to correct for head movement. We included six movement parameters (translation: $\mathrm{x}, \mathrm{y}, \mathrm{z}$; rotation: pitch, roll, yaw) in the statistical model. We normalised the functional images to a standard Montreal Neurological Institute EPI template and we then spatially smoothed them using a $4-\mathrm{mm}$, full-width, half-maximum isotropic Gaussian kernel.

\section{Regions of interest analysis}

We obtained parameter estimates of signal intensity from structurally defined amygdala to assess genotype treatment interaction on emotional processes. We identified the amygdala using the Automated Anatomical Labeling (AAL) atlas (AAL templates) and we selected it using the Wake Forest University School of Medicine PickAtlas software toolbox. We subjected the parameter estimates of signal intensity in the left and right amygdala to repeated-measures analyses of variance (ANOVA) with genotype $(s / s \quad v .1 / 1)$ as a between-subjects variable, and treatment (citalopram $v$. placebo) and emotion (fearful $v$. neutral facial expressions or happy $v$. neutral facial expressions) as between-subjects independent variables. To further clarify whether differential SSRI effects on amygdala activity were driven by neural response to emotional or neutral facial expressions, we calculated the contrast value related to fearful, happy and neutral facial expressions $v$. fixation cross, as used in previous studies. ${ }^{35}$ We then subjected these contrast values to genotype treatment ANOVAs for fearful, happy and neutral facial expressions, respectively. We included the treatment (citalopram $v$. placebo) sequence as a covariate in region of interest (ROI) and whole-brain analyses to control for any potential effect of treatment order. We also conducted analyses that included treatment order as a betweensubject variable in the ANOVA and this did not show significant treatment order effects.

\section{Whole-brain analysis}

We used a two-level, hierarchical, random-effects model for whole-brain analysis. At the individual level, we modelled the onset and duration of each block using a general linear model for five conditions: fearful, happy, neutral, scrambled facial expression and fixation cross. We used a box-car function to convolve with a canonical haemodynamic response in each condition. The contrasts of 'fearful $v$. neutral' and 'happy $v$. neutral' identified brain regions responsive to positive and negative emotional facial expressions, respectively. We then performed a whole-brain factorial design to reveal the genotype treatment interaction on the neural response to fearful or happy facial expressions. Significant activations were identified using a threshold of $P<0.05$ (topological false discovery rate (FDR) corrected for multiple comparisons correction).

\section{Results}

\section{Behavioural results}

Response accuracy was high in the repetition-detection tasks during scanning (mean accuracy $>95 \%$ ). We subjected response accuracy and reaction times to 2 (emotion: fearful $v$. neutral facial expressions, or happy $v$. neutral facial expressions) $\times 2$ (treatment: citalopram $v$. placebo $) \times 2$ (genotype: $s / s v$. 1/l) ANOVAs, but did not show any significant effect $(P>0.05)$.

We subjected self-reported mood changes from pre- to postscan to a 2 (treatment: citalopram $v$. placebo) $\times 2$ (genotype: s/s v. 1/l) ANOVA. This did not show any significant effect $(F<1$, Table DS1), suggesting that citalopram treatment did not have an effect on the participants' mood. Harm avoidance scores did not differ between $\mathrm{s} / \mathrm{s}$ and $1 / 1$ homozygotes (18.2 v. 16.0, $t(44)=1.07, P=0.291)$. Participants rated fearful faces as less fearful after citalopram than placebo administration $(F(1,43)=$ 5.44, $P=0.024)$, but this effect did not differ significantly between $\mathrm{s} / \mathrm{s}$ and $1 / 1$ homozygotes $(F(1,43)=2.44, P=0.126$, Fig. DS1). Relative to $1 / 1$ homozygotes, $s / s$ homozygotes reported greater emotional intensity of happy facial expressions $(F(1,43)=7.30$, $P=0.010)$, but neither the main effect of treatment nor its interaction with genotype was significant $(P>0.35)$.

\section{Functional magnetic resonance imaging}

Region of interest analyses of amygdala response to fearful $v$. neutral facial expressions in the placebo session revealed significantly stronger left amygdala response in $s / s$ than $1 / 1$ 


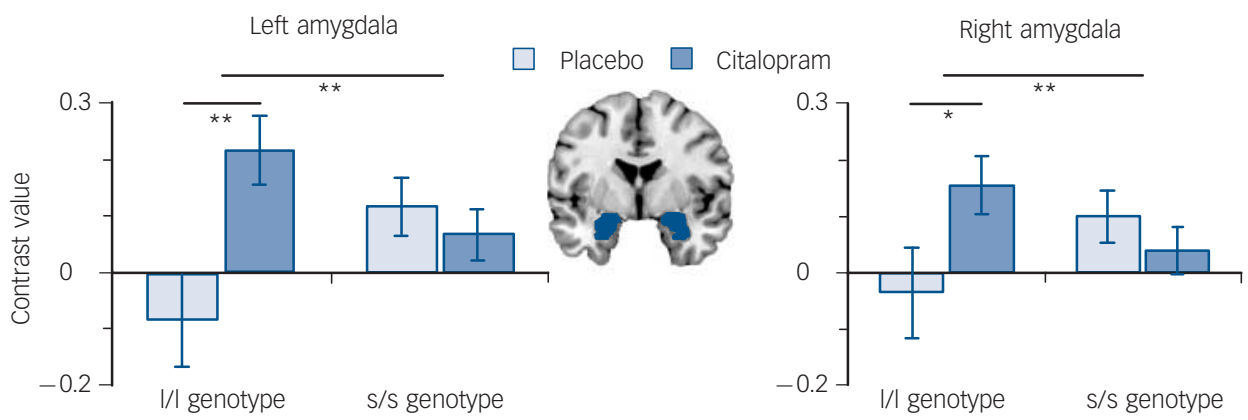

|/| Placebo

I/I Citalopram

s/s Placebo

s/s Citalopram

(b)

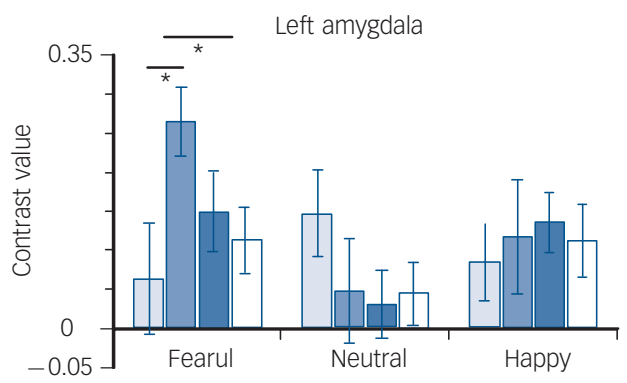

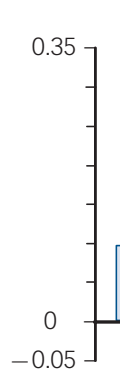

Right amygdala
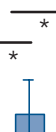

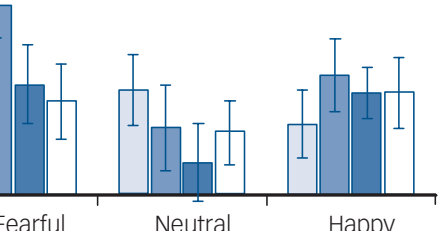

Fearful Neutral Happy

(C)

I/I genotype (Citalopram/Placebo) v. s/s genotype (Citalopram/Placebo)

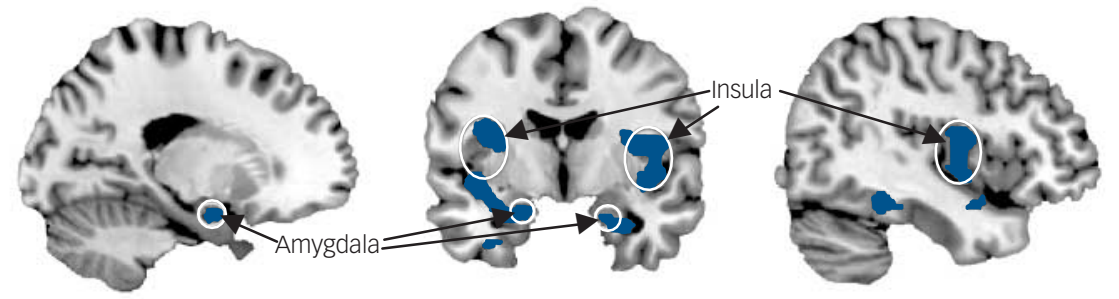

Fig. 2 Brain imaging results. (a) Contrast values of fearful v. neutral faces of the left and right amygdala. (b) Contrast values of the left and right amygdala response to fearful, neutral and happy faces compared to fixation. (c) Whole-brain treatment $\times$ genotype interaction on the contrast of fearful $v$. neutral faces. This revealed significant activations in the left and right amygdala and insula (*P<0.05, $* \star P<0.01)$.

genotype groups $(F(1,43)=4.15, P=0.048$, Fig. 2a), replicating previous findings. ${ }^{36,37}$ We assessed the differential citalopram effects on amygdala response to fearful $v$. neutral facial expressions in the two genotypes by conducting a 2 (emotion: fearful $v$. neutral $) \times 2$ (treatment: citalopram $v$. placebo $) \times 2$ (genotype: $s / s$ v. 1/l) ANOVA of parameter estimates of signal intensity. This revealed a significant main effect of emotion in both left and right amygdala $(F(1,43)=5.40$ and $4.12, P=0.025$ and 0.048$)$, indicating greater amygdala responses to fearful than neutral facial expressions. Similar to previous findings, ${ }^{11,12,20}$ we found a significant main effect of treatment on left amygdala response $(F(1,43)=5.14$, $P=0.028$ ), indicating that, relative to placebo, acute administration of citalopram in healthy individuals increased activation in the left amygdala to fearful $v$. neutral facial expressions.

Notably, there was a significant three-way interaction of emotion $\times$ treatment $\times$ genotype on response in both left and right amygdala $(F(1,43)=10.47$ and $8.42, P=0.002$ and 0.006 , Fig. $2 a)$, suggesting that acute administration of citalopram increased response in the left and right amygdala to fearful $v$. neutral faces in the $1 / 1$ genotype (left: $(F(1,21)=10.87, P=0.003$; right: $F(1,21)=4.57, P=0.045)$ but did not significantly affect amygdala response in the s/s genotype (left: $F(1,21)=1.53, P=0.229$; right: $F(1,21)=1.42, P=0.248)$. To further assess whether the three-way interaction was driven by citalopram effect on amygdala response to fearful or neutral facial expressions, we examined the treatment $\times$ genotype interaction on amygdala response in response to fearful and neutral facial expressions ( $v$. fixation), respectively. We found significant treatment $\times$ genotype interactions on amygdala response to fearful facial expressions (left: $F(1,43)=5.48, P=0.024$; right: $F(1,43)=4.14, P=0.048$, Fig. $2 b)$, but not to neutral facial expressions $(P>0.2)$. Thus, the differential citalopram effects on amygdala response in the $1 / 1$ and s/s genotypes arose from amygdala response to fearful rather than neutral facial expressions. Similar analyses of amygdala response to happy facial expressions failed to show any significant effect $(P>0.1$, Fig. $2 \mathrm{~b})$.

To further confirm the distinct citalopram effects on amygdala response between the two genotypes of 5-HTTLPR, we conducted a whole-brain analysis to test the interaction between genotype (s/s $v .1 / \mathrm{l})$ and treatment (citalopram $v$. placebo) on the contrast of fearful $v$. neutral facial expressions. This revealed significant interactions of genotype $\times$ treatment in left and right amygdala response (left: $-18 /-2 /-20, \mathrm{~T}=2.98$; right: $30 /-4 /-28$, $\mathrm{T}=3.13, P<0.05$, topological FDR corrected), further confirming the differential citalopram effects on amygdala response. The whole-brain analysis also revealed significant interactions of genotype $\times$ treatment in left and right insula response (left: 
$-34 /-12 / 16, \quad \mathrm{~T}=3.91 ; \quad$ right: $40 /-4 / 12, \quad \mathrm{~T}=3.35, \quad P<0.05$, topological FDR corrected; Fig. 2c), suggesting variations of citalopram effects on insula response as a function of 5-HTTLPR polymorphism (Fig. 3). A similar whole-brain analysis of the contrast of happy $v$. neutral facial expressions did not show any significant brain activation.

\section{Discussion}

Current research has focused on elucidating the neural substrates underlying the heterogeneity of the acute effects of SSRIs in healthy volunteers. We have provided pharmacogenetic and neuroimaging evidence that 5-HTTLPR polymorphism modulates the acute effects of citalopram on neural activity related to emotion processing. Specifically, we found that the acute administration of citalopram ( $v$. placebo) increased amygdala and insula activity in the $1 / 1$ but not the $\mathrm{s} / \mathrm{s}$ genotype of 5-HTTLPR. The genotype differences in the acute effects of an SSRI were evident in the neural responses to fearful but not happy facial expressions, indicating that such genotype differences do not reflect the non-specific effects of citalopram, such as changes in arousal or drowsiness. Our results indicate that an individual's genetic structure may influence the acute effects of citalopram on neural response to negative emotions and that 5-HTT is a key molecular transporter that contributes to the differential neural responses to negative emotions in healthy adults. Our findings provide a possible neurogenetic mechanism for understanding the previous inconsistent results regarding how the acute administration of SSRIs modulates neural responses to negative emotions.

Self-reported mood was not influenced by 5-HTTLPR genotype or citalopram administration. Therefore, 5-HTTLPR modulation of the effect of citalopram on amygdala response cannot be simply attributed to general mood changes. This is consistent with a cognitive neuropsychological model of SSRI action that emotional processes are sensitive to early SSRI-induced changes in the absence of mood variation. ${ }^{4,5}$ The post-scan rating scores suggested decreased emotion-intensity rating of fearful facial expressions after citalopram $v$. placebo treatment. However, this effect was the same in the s/s and 1/l genotypes. Similarly, 7 days of SSRI treatment decreased amygdala response to fearful facial expressions but failed to induce significant changes in clinical depression ratings. ${ }^{38}$ Thus amygdala response may be more sensitive to how 5-HTTLPR modulates the acute administration of citalopram and thus provides a neurobiological index of genetic influences on the effect of SSRIs.

The 5-HTTLPR also modulated the acute effects of citalopram on insula response to fearful facial expressions. The insula plays a key role in how emotions are processed, such as detection of emotional salience ${ }^{39}$ and anticipation of emotional stimuli. ${ }^{40}$ Chronic SSRI treatment attenuates insula activation during perception of emotional facial expressions ${ }^{41}$ and during anticipation of emotional stimuli ${ }^{42}$ in healthy volunteers. The acute administration of an SSRI, however, increased insula response. ${ }^{13}$ Our findings suggest that 5 -HTT plays a pivotal role in producing the differential insula response in the $s / s$ and $1 / 1$

(a)
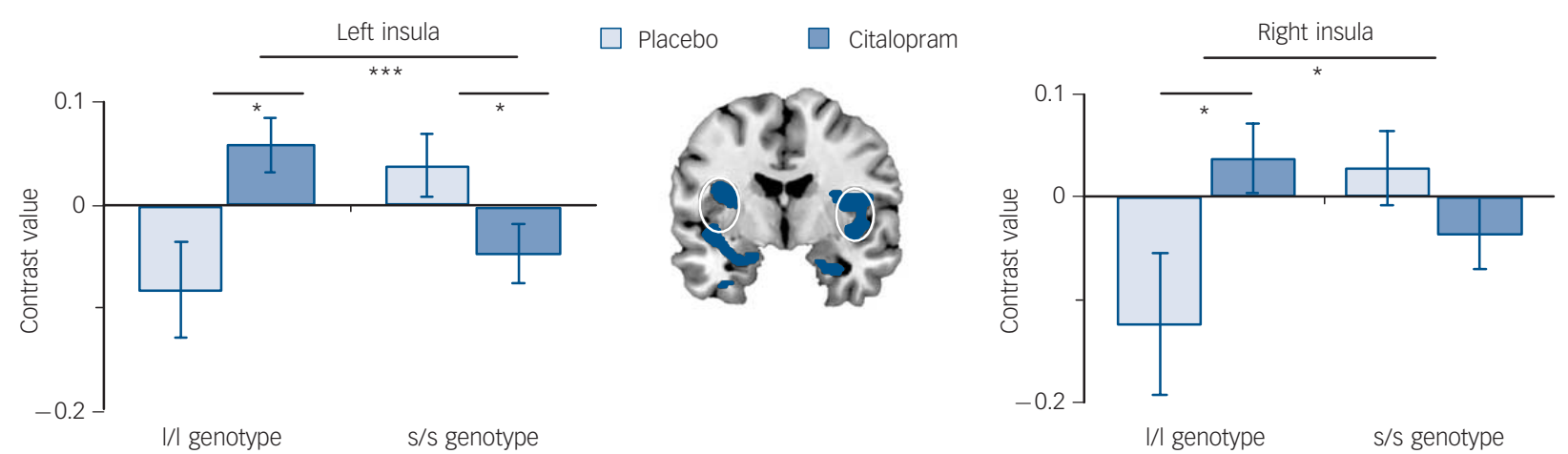

(b)
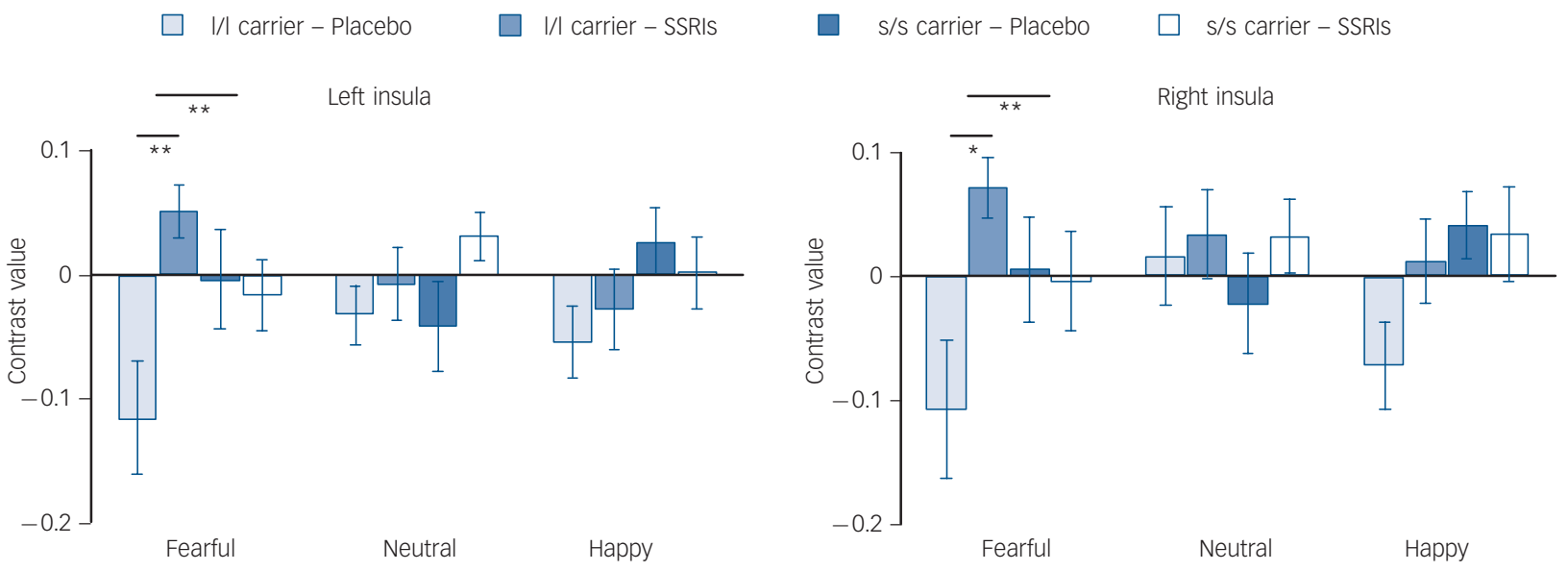

s/s carrier - Placebo

S/S carrier - SSRIS

Fig. 3 Contrast values calculated from the left and right insula. (a) Contrast values of fearful $v$. neutral faces in the left and right insula revealed by the genotype treatment interaction in the whole-brain analysis; (b) contrast values of faces $v$. fixation in the left and right insula. The effect of citalopram was significant only for fearful faces $\left({ }^{*} P<0.05,{ }^{*} P<0.01 ;{ }^{*} * P<0.001\right)$. SSRI: selective serotonin reuptake inhibitor. 
genotypes. Taken together, our results suggest that the 5-HTTLPR genotype may influence the effect of citalopram on neural response to negative emotions in different nodes (e.g. amygdala and insula) of the emotional neural network in a similar vein.

\section{Implications}

Our pharmacogenetic and neuroimaging findings have important implications. First, whereas amygdala response is modulated by the intensity of both fearful and happy facial expressions, ${ }^{43}$ the acute administration of an antidepressant in healthy volunteers did not show convergent response to positive emotions. ${ }^{20}$ Some studies have reported that the acute administration of an SSRI facilitates recognition of happy facial expressions and increased amygdala response to happy facial expressions. ${ }^{10,18}$ Other studies have failed to show significant SSRI effects on neural or behavioural responses to happy facial expressions. ${ }^{9,15,16,19}$ Similarly, the acute administration of citalopram did not change subjective feelings or amygdala response related to happy facial expressions in both genotypes in the current study. Thus, 5-HTT may play a less important role in affective and neural responses to positive (relative to negative) emotion. Anhedonia (i.e. the inability to obtain pleasure from natural rewards and decreased interest in most activities) and negative emotional bias are two core features of depression. Depressed patients tend to interpret happy facial expressions as neutral, fail to experience pleasure from activities they previously experienced as rewarding and lack reward-motivated behaviours. By contrast, they pay more attention to negative emotions, remember negative affective materials better and are more likely to classify ambiguous/neutral facial expressions as negative. ${ }^{20}$ Thus our findings suggest that citalopram may have distinct therapeutic implications for the treatment of different symptoms in depressed patients. Alternatively, the lack of 5-HTTLPR modulation of the acute effects of an SSRI on amygdala response to happy facial expressions may be due to the absence of amygdala activation to happy facial expressions in our experiment. This should be clarified in future research.

Second, the early changes in emotional processing after a single SSRI dose contribute to later mood improvements. ${ }^{3-5}$ Thus, understanding the neural mechanisms underlying the acute effects of SSRIs has important implications for long-term treatment. Our findings of distinct acute effects of an SSRI on emotion-related neural activity in $s / s$ and $1 / 1$ homozygotes suggest that one's genetic structure should be considered when treating mood disorders with SSRIs. However, it remains unknown whether and how 5-HTTLPR modulates the chronic SSRI effects that lead to decreased response to negative emotions in both healthy controls and depressed patients. ${ }^{20}$ Pharmacogenetic studies of antidepressant response reported better responses of the 'l' variant (compared with the 's' variant) of 5-HTTLPR to SSRIs in mood disorder patients. ${ }^{25,44,45}$ Our fMRI results of differential acute effects of citalopram on amygdala and insula response in $1 / 1$ and $\mathrm{s} / \mathrm{s}$ homozygotes are consistent with the distinct SSRI effects on clinical assessment of improvement in patients with major depression. ${ }^{24}$ These results together suggest more pronounced SSRI effects in $1 / 1$ than in s/s homozygotes and are consistent with the findings of increased 5-HTT expression ${ }^{26}$ and increased 5-HT neurotransmission after acute blockade of 5 -HT reuptake ${ }^{27}$ in 1/1 homozygotes than ' $s$ ' carriers. Future research on genetic differences in chronic SSRI treatment may open an avenue for prescribing personalised antidepressants.

Third, our finding that citalopram increased amygdala response to fearful facial expressions in 1/1 homozygotes suggests a more anxiogenic action of acute SSRI treatment in this group.
This seems counterintuitive given that mood disorder patients with the 'l' variant (compared with the 's' variant) of 5-HTTLPR showed a better SSRI response. ${ }^{25,44,45}$ However, opposite early effects of SSRIs in depression have been reported in clinical treatment. The acute administration of SSRIs may increase anxiety in some mood disorder patients. ${ }^{46}$ Our findings thus provide a potential neurogenetic explanation for the opposite early effect of SSRIs in clinical trials. Future research should address whether and how early SSRI-induced changes in emotional processes can predict long-term treatment efficacy and whether one's genetic structure modulates the early and chronic effects of SSRIs in a similar way. Future research should also address whether such increased amygdala response to fearful facial expressions is dosedependent; i.e. whether a lower or higher dose of citalopram could modulate this effect.

Finally, although the ' $s$ ' allele frequency is different between Asian and White populations, ${ }^{47}$ to date, we know little about whether the ' $\mathrm{s}$ ' allele frequency in a population is associated with a specific pattern of 5-HTTLPR modulation of brain activity. Previous research showed an association between the ' $s$ ' allele and hyperactivity in the amygdala mainly in White populations. ${ }^{36,37,48}$ Our study involving Chinese healthy volunteers in the placebo group also showed stronger amygdala response to fearful ( $v$. neutral) facial expressions in the $s / s$ than $1 / 1$ genotype group. This is similar to the observation of 5-HTTLPR effects on amygdala response in White populations. ${ }^{36,37,48}$ In addition, we found that $1 / 1$ compared to $\mathrm{s} / \mathrm{s}$ homozygotes tended to show greater amygdala response in the contrast of neutral facial expressions $v$. fixation in the placebo condition, replicating the previous findings in White populations. ${ }^{35}$ These results suggest similar 5-HTTLPR effects on amygdala activity regardless of the difference in the ' $s$ ' allele frequency in populations. Pharmacogenetic studies have reported inconsistent 5-HTTLPR effects on antidepressant drug response in White and Asian groups experiencing major depression. ${ }^{45}$ However, the improved response to SSRIs in the 'l' variant of 5 -HTTLPR seemed to be independent of ethnic differences, ${ }^{25}$ which may be confirmed by future research.

\section{Limitations}

A potential limitation of the present study was the sample size. To examine the power of our sample size, we calculated the effect size and conducted a power analysis. These analyses suggested that our study yielded a large effect size and appropriate sample size (see online supplementary material for details). We also reviewed published studies that examined 5-HTTLPR genotype differences on brain activity and studies that examined antidepressant effects on emotional neural responses. According to a recent meta-analysis, 26 fMRI studies examined 5-HTTLPR genotype differences in emotional reactivity. ${ }^{48}$ The mean sample size of these 26 studies was 38. Mode of sample size was 28 . Our recent meta-analysis summarised antidepressant effects on emotional responses. ${ }^{20}$ Twenty-one fMRI studies used between-subjects treatment (the same design as the current study) to examine antidepressant effects on brain responses to emotions in healthy participants. The average sample size of these 21 studies was 15 . Considering the effect size of the current work and comparing the sample size of published studies, the sample size of our study allowed us to reach a conclusion (albeit with caution) on 5-HTTLPR genotype differences in SSRI effects on emotional neural activity.

Another possible limitation was that only men were recruited for the current study. This did not allow us to test whether 5-HTTLPR modulates the SSRI effect on amygdala responses in men and women in a similar fashion. Previous research has revealed similar 5-HTTLPR effects on the reactivity of the 
amygdala to affective stimuli in men and women. ${ }^{37} \mathrm{~A}$ recent study showed that acute treatment with escitalopram induced changes in response to negative stimuli in the left amygdala and this effect decreased with an increasing number of ' 1 ' alleles of 5-HTTLPR in healthy White women. ${ }^{49}$ However, it is difficult to compare our results with previous findings because of differences in participants' ethnicity, subtypes of SSRIs and stimuli/paradigms. Thus, whether there is a 5 -HTTLPR $\times$ gender interaction of SSRI treatment is still an open question.

The current study did not find any significant effect of treatment or genotype on participants' behavioural performances in the repetition-detection tasks during scanning. The lack of significant effect of treatment or genotype on behavioural performance may be driven by: (a) a ceiling effect of behavioural responses, since the repetition-detection task was easy; (b) a limited number of response-required trials, i.e. eight responserequired trials for each stimulus category; or (c) the implicit emotional task used in our study, as antidepressant treatment produced distinct effects on implicit and explicit emotional processes. ${ }^{20}$ A more sensitive and direct behavioural measure may reveal the treatment and genotype effect on behavioural performance.

\section{A neurogenetic mechanism in SSRI response}

While there has been increasing interests in pharmacogenetic studies to identify predictors of drug efficacy, ${ }^{50,51}$ our findings of 5-HTTLPR modulation of the effects of SSRIs shed new light on genetic constraints on the acute effects of SSRIs on neural activity underlying emotional processing. Our results suggest possible functional mechanisms by which acute administration of SSRIs may exert effects on emotional responses. Our findings suggest that the 'small' effect size of psychometric outcome measures of drug response $e^{50,51}$ may be improved by using a pharmacogenetic imaging approach that can help to identify intermediate endophenotypes of drug response.

Yina Ma, PhD, Department of Psychology, Peking University, China, and Lieber Yina Ma, PhD, Department of Psychology, Peking University, China, and Lieber
Institute for Brain Development, Johns Hopkins University School of Medicine, Baltimore, USA; Bingfeng Li, BS, Peking-Tsinghua Center for Life Sciences at Schoo of Life Sciences and PKU-IDG/MCGovern Institute for Brain Research, Peking University, Beijing, China; Chenbo Wang, PhD, Department of Psychology and PKU-IDG/McGovern Institute for Brain Research, Peking University, Beijing, China; Wenxia Zhang, PhD, Peking-Tsinghua Center for Life Sciences at School of Life Sciences and PKU-IDG/McGovern Institute for Brain Research, Peking University, Beijing, China; Yi Rao, PhD, Peking-Tsinghua Center for Life Sciences at School of Life Beijing, China; YI Rao, PhD, Peking-Tsinghua Center for Life Sciences at School of
Sciences and PKU-IDG/McGovern Institute for Brain Research, Peking University, Beijing, China; Shihui Han, PhD, Department of Psychology and PKU-IDG/McGovern Institute for Brain Research, Peking University, Beijing, China.

Correspondence: Yina Ma, Lieber Institute for Brain Development, 855 North Wolfe Street, Baltimore, MD 21205, USA, or Shihui Han, Department of Psychology and PKU-IDG, MCGovern Institute for Brain Research, Peking University, 5 Yiheyuan Road, Beijing 100871,China. Email: yina.ma@libd.org or shan@pku.edu.cn

First received 17 Apr 2014, final revision 11 Sep 2014, accepted 15 Sep 2014

\section{Funding}

This work was supported by the National Natural Science Foundation of China (Project 91332125, 31421003, 31470986), the National Basic Research Program of China (973 Program 91332125, 31421003, 31470986), the National Basic Research Program of China (973 Program
2010CB833901, 2010CB833903), Beijing Municipal Natural Science Foundation (Z111107067311058), and the Ministry of Education of China (Project 20130001110049).

\section{Acknowledgements}

We thank Siyang Luo, Yi Liu, Yifan Zhang, Zhenhao Shi, Xiangyu Zuo, Dian Yu, Rui Sun, Xiaoyang Li for their help with recruitment of participants. We thank Sook-Lei Liew for proofreading of this paper.

\section{References}

1 Meyer JH, Wilson AA, Sagrati S, Hussey D, Carella A, Potter WZ, et al. Serotonin transporter occupancy of five selective serotonin reuptake inhibitors at different doses: an [11C]DASB positron emission tomography study. Am J Psychiatry 2004; 161: 826-35.

2 Bosker FJ, Cremers TI, Jongsma ME, Westerink BHC, Wikström HV, den Boer JA. Acute and chronic effects of citalopram on postsynaptic 5-hydroxytryptamine $(1 \mathrm{~A})$ receptor-mediated feedback: a microdialysis study in the amygdala. J Neurochem 2001; 76: 1645-53.

3 Taylor MJ, Freemantle N, Geddes JR, Bhagwagar Z. Early onset of selective serotonin reuptake inhibitor antidepressant action: systematic review and meta-analysis. Arch Gen Psychiatry 2006; 63: 1217-23.

4 Harmer CJ, Goodwin GM, Cowen PJ. Why do antidepressants take so long to work? A cognitive neuropsychological model of antidepressant drug action. Br J Psychiatry 2009; 195: 102-8.

5 Pringle A, Browning M, Cowen PJ, Harmer CJ. A cognitive neuropsychological model of antidepressant drug action. Prog Neuropsychopharmacol Biol Psychiatry 2011; 35: 1586-9.

6 Drevets WC. Neuroimaging studies of mood disorders. Biol Psychiatry 2000; 48: 813-29.

7 Ketter TA, Kimbrell TA, George MS, Dunn RT, Speer AM, Benson BE, et al. Effects of mood and subtype on cerebral glucose metabolism in treatmentresistant bipolar disorder. Biol Psychiatry 2001; 49: 97-109.

8 McKie S, Del-Ben C, Elliott R, Williams S, del Vai N, Anderson I, et al. Neuronal effects of acute citalopram detected by pharmacoMRI. Psychopharmacology (Berl) 2005; 180: 680-6.

9 Brühl AB, Kaffenberger T, Herwig U. Serotonergic and noradrenergic modulation of emotion processing by single dose antidepressants. Neuropsychopharmacology 2010; 35: 521-33.

10 Macoveanu J, Rowe JB, Hornboll B, Elliott R, Paulson OB, Knudsen GM, et al. Playing it safe but losing anyway-Serotonergic signaling of negative outcomes in dorsomedial prefrontal cortex in the context of risk-aversion. Eur Neuropsychopharmacol 2013; 23: 919-30.

11 Grady CL, Siebner HR, Hornboll B, Macoveanu J, Paulson OB, Knudsen GM Acute pharmacologically induced shifts in serotonin availability abolish emotion-selective responses to negative face emotions in distinct brain networks. Eur Neuropsychopharmacol 2013; 23: 368-78.

12 Bigos KL, Pollock BG, Aizenstein HJ, Fisher PM, Bies RR, Hariri AR. Acute 5 -HT reuptake blockade potentiates human amygdala reactivity. Neuropsychopharmacology 2008; 33: 3221-5.

13 Anderson IM, Del-Ben CM, Mckie S, Richardson P, Williams SR, Elliott R, et al. Citalopram modulation of neuronal responses to aversive face emotions: a functional MRI study. Neuroreport 2007; 18: 1351-5.

14 Anderson IM, Juhasz G, Thomas E, Downey D, McKie S, Deakin JF, et al. The effect of acute citalopram on face emotion processing in remitted depression: a pharmacoMRI study. Eur Neuropsychopharmacol 2011; 21 140-8.

15 Del-Ben CM, Deakin JF, McKie S, Delvai NA, Williams SR, Elliott R, et al. The effect of citalopram pretreatment on neuronal responses to neuropsychological tasks in normal volunteers: an FMRI study. Neuropsychopharmacology 2005; 30: 1724-34.

16 Murphy SE, Norbury R, O'Sullivan U, Cowen PJ, Harmer CJ. Effect of a single dose of citalopram on amygdala response to emotional faces. Br J Psychiatry 2009; 194: 535-40.

17 Takahashi H, Yahata N, Koeda M, Takano A, Asai K, Suhara T, et al. Effects of dopaminergic and serotonergic manipulation on emotional processing: a pharmacological fMRI study. Neuroimage 2005; 27: 991-1001.

18 Outhred T, Das P, Felmingham KL, Bryant RA, Nathan PJ, Malhi GS, et al. Impact of acute administration of escitalopram on the processing of emotional and neutral images: a randomized crossover fMRI study of healthy women. J Psychiatry Neurosci 2014; 39: 267-75.

19 Brühl $A B$, Jäncke $L$, Herwig U. Differential modulation of emotion processing brain regions by noradrenergic and serotonergic antidepressants. Psychopharmacology (Berl) 2011; 216: 389-99.

20 Ma Y. Neuropsychological mechanism underlying antidepressant effect: a systematic meta-analysis. Mol Psychiatry 2014. DOI: 10.1038/mp.2014.24.

21 Weinshilboum R. Inheritance and drug response. N Engl J Med 2003; 348: 529-37.

22 Heils A, Teufel A, Petri S, Stöber G, Riederer P, Bengel D, et al. Allelic variation of human serotonin transporter gene expression. J Neurochem 1996; 66: 2621-4.

23 Canli T, Lesch KP. Long story short: the serotonin transporter in emotion regulation and social cognition. Nat Neurosci 2007; 10: 1103-9. 
24 Smeraldi E, Zanardi R, Benedetti F, Di Bella D, Perez J, Catalano M. Polymorphism within the promoter of the serotonin transporter gene and antidepressant efficacy of fluvoxamine. Mol Psychiatry 1998; 3: 508-11.

25 Serretti A, Kato M, De Ronchi D, Kinoshita T. Meta-analysis of serotonin transporter gene promoter polymorphism (5-HTTLPR) association with selective serotonin reuptake inhibitor efficacy in depressed patients. Mol Psychiatry 2007; 12: 247-57.

26 Lesch KP, Bengel D, Heils A, Sabol SZ, Greenberg BD, Petri S, et al. Association of anxiety-related traits with a polymorphism in the serotonin transporter gene regulatory region. Science 1996; 274: 1527-31.

27 Whale R, Quested DJ, Laver D, Harrison PJ, Cowen PJ. Serotonin transporte (5-HTT) promoter genotype may influence the prolactin response to clomipramine. Psychopharmacology (Berl) 2000; 150: 120-2.

28 Crockett MJ, Clark L, Hauser MD, Robbins TW. Serotonin selectively influences moral judgment and behavior through effects on harm aversion. Proc Natl Acad Sci U S A 2010; 107: 17433-8.

29 Nandam LS, Hester R, Wagner J, Cummins TD, Garner K, Dean AJ, et al. Methylphenidate but not atomoxetine or citalopram modulates inhibitory control and response time variability. Biol Psychiatry 2011; 69: 902-4.

30 Penttilä J, Syvälahti E, Hinkka S, Kuusela T, Scheinin H. The effects of amitriptyline, citalopram and reboxetine on autonomic nervous system. A randomised placebo-controlled study on healthy volunteers. Psychopharmacology (Berl) 2001; 154: 343-9.

31 Jiang T, Rong Z, Xu Y, Chen B, Xie Y, Chen C, et al. Pharmacokinetics and bioavailability comparison of generic and branded citalopram $20 \mathrm{mg}$ tablets: an open-label, randomized-sequence, two-period crossover study in healthy Chinese CYP2C19 extensive metabolizers. Clin Drug Investig 2013; 33: 1-9.

32 Cloninger CR, Svrakic DM, Przybeck TR. A psychobiological model of temperament and character. Arch Gen Psychiatry 1993; 50: 975-90.

33 Watson D, Clark LA, Tellegen A. Development and validation of brief measures of positive and negative affect: the PANAS scales. J Pers Soc Psychol 1988; 54: 1063-70.

34 Gong $X$, Huang $Y-X$, Wang $Y$, Luo Y-J. Revision of the Chinese Facial Affective Picture System. Chin Ment Health J 2011; 25: 40-6.

35 Heinz A, Smolka MN, Braus DF, Wrase J, Beck A, Flor H, et al. Serotonin transporter genotype (5-HTTLPR): effects of neutral and undefined conditions on amygdala activation. Biol Psychiatry 2007; 61: 1011-14.

36 Hariri AR, Mattay VS, Tessitore A, Kolachana B, Fera F, Goldman D, et al. Serotonin transporter genetic variation and the response of the human amygdala. Science 2002; 297: 400-3.

37 Hariri AR, Drabant EM, Munoz KE, Kolachana BS, Mattay VS, Egan MF, et al. A susceptibility gene for affective disorders and the response of the human amygdala. Arch Gen Psychiatry 2005; 62: 146-52.
38 Godlewska BR, Norbury R, Selvaraj S, Cowen PJ, Harmer CJ. Short-term SSRI treatment normalises amygdala hyperactivity in depressed patients. Psychol Med 2012; 42: 2609-17.

39 Craig $A D$. How do you feel? Interoception: the sense of the physiological condition of the body. Nat Rev Neurosci 2002; 3: 655-66.

40 Simmons A, Matthews SC, Stein MB, Paulus MP. Anticipation of emotionally aversive visual stimuli activates right insula. Neuroreport 2004; 15: 2261-5.

41 Arce E, Simmons AN, Lovero KL, Stein MB, Paulus MP. Escitalopram effects on insula and amygdala BOLD activation during emotional processing. Psychopharmacology (Berl) 2008; 196: 661-72.

42 Simmons AN, Arce E, Lovero KL, Stein MB, Paulus MP. Subchronic SSRI administration reduces insula response during affective anticipation in healthy volunteers. Int J Neuropsychopharmacol 2009; 12: 1009-20.

43 Morris JS, Frith CD, Perrett DI, Rowland D, Young AW, Calder AJ, et al. A differential neural response in the human amygdala to fearful and happy facial expressions. Nature 1996; 383: 812-5.

44 Smits KM, Smits L, Schouten JS, Stelma FF, Nelemans P, Prins MH. Influence of SERTPR and STin2 in the serotonin transporter gene on the effect of selective serotonin reuptake inhibitors in depression: a systematic review. Mol Psychiatry 2004; 9: 433-41.

45 Malhotra AK, Murphy GM Jr, Kennedy JL. Pharmacogenetics of psychotropic drug response. Am J Psychiatry 2004; 161: 780-96.

46 Mir S, Taylor D. The adverse effects of antidepressants. Curr Opin Psychiatry 1997: 10: 88-94.

47 Kunugi $\mathrm{H}$, Hattori $\mathrm{M}$, Kato $\mathrm{T}$, Tatsumi $\mathrm{M}$, Sakai $\mathrm{T}$, Sasaki $\mathrm{T}$, et al. Serotonin transporter gene polymorphisms: ethnic difference and possible association with bipolar affective disorder. Mol Psychiatry 1997; 2: 457-62.

48 Murphy SE, Norbury R, Godlewska BR, Cowen PJ, Mannie ZM, Harmer CJ, et al. The effect of the serotonin transporter polymorphism (5-HTTLPR) on amygdala function: a meta-analysis. Mol Psychiatry 2013; 18: 512-20.

49 Outhred T, Das P, Dobson-Stone C, Felmingham KL, Bryant RA, Nathan PJ, et al. The impact of 5-HTTLPR on acute serotonin transporter blockade by escitalopram on emotion processing: preliminary findings from a randomised, crossover fMRI study. Aust N Z J Psychiatry 2014; 48 1115-25.

50 Rabl U, Scharinger C, Müller M, Pezawas L. Imaging genetics: implications for research on variable antidepressant drug response. Expert Rev Clin Pharmacol 2010; 3: 471-89.

51 Malhotra AK, Zhang JP, Lencz T. Pharmacogenetics in psychiatry: translating research into clinical practice. Mol Psychiatry 2012; 17: 760-9. 